\title{
A probabilistic indirect adaptive control for systems with input-dependent noise
}

\author{
Randa Herzallah \\ FET \\ Al-Balqa' Applied University, Jordan \\ Email:herzallah.r@gmail.com
}

SUMMARY

A probabilistic indirect adaptive controller is proposed for the general nonlinear multivariate class of discrete time system. The proposed probabilistic framework incorporates input-dependent noise prediction parameters in the derivation of the optimal control law. Moreover, because noise can be nonstationary in practice, the proposed adaptive control algorithm provides an elegant method for estimating and tracking the noise. For illustration purposes, the developed method is applied to the affine class of nonlinear multivariate discrete time systems and the desired result is obtained: the optimal control law is determined by solving a cubic equation and the distribution of the tracking error is shown to be Gaussian with zero mean. The efficiency of the proposed scheme is demonstrated numerically through the simulation of an affine nonlinear system.

Copyright (c) 2010 John Wiley \& Sons, Ltd.

1. Introduction

It can generally be assumed that any system in the real world has some degree of uncertainty, which is a consequence of the uncertainty due to the intrinsic nonlinear dynamic of the system, noise and

\footnotetext{
$\dagger$ Please ensure that you use the most up to date class file, available from the ACS Home Page at http://www.interscience.wiley.com/jpages/0890-6327/
} 
randomness in data and approximate model parameters. It is therefore, a necessary requirement for any controller to be able to estimate and incorporate such uncertainty. The classical way of solving the problem of uncertain parameters is to use adaptive filters where not only the dynamic behavior of the system is estimated but also the noise statistics [11,14]. The most general approach for solving the noise adaptive filtering was the Bayesian approach $[13,14,19]$. Several applications of the Bayesian method have been discussed in the literature, examples are: the control of the basic oxygen furnace [10], traffic flow forecasting [20] and the estimation of the river reach time delay [21]. However, for complex control problems it is insufficient to estimate parameters uncertainty only. Models uncertainty should also be estimated and incorporated in deriving the optimal control law. To deal with the high levels of uncertainty in modeling and estimation and the growing complexity of control systems, a number of controller algorithms have been proposed. Three groups of the so far known developed control methods are: i) the shape control of the closed loop probability density function [9]; ii) the control of the output probability density functions $[22,23]$; and iii) the control of the tracking error probability density functions [7]. In the first group the controller is designed such as to minimize the distance between the actual closed loop probability density function and a predefined desired density function. In that method, all required probability density functions are assumed to exist. In the second group the objective of the controller design is to find a control input which makes the shape of the measured output probability density function follows a given distribution. The objective of the controller in the third method however, is to characterize the uncertainty of the tracking error for general nonlinear systems. It estimates the distribution of the inverse controller which makes the average tracking error equal to zero. The problem in [7] is formulated from the Bayesian decision theoretic viewpoint which is shown to be a general framework to solve stochastic estimation and control problems [8]. Despite the theoretical attractiveness of the first and second groups, they treat uncertainty as a nuisance or perturbation which does not affect the derivation of the optimal control law. In other words, uncertainty has been assumed to be input-independent and consequently they did not contribute to the derivation of the optimal control law. The third design group on the other hand considers input-dependent uncertainty in the forward model. No allowance is made for uncertainty of the tracking error.

The method proposed in [7] is constrained to the case where the noise variance of the tracking error represented by the hyperparameter of the tracking error distribution was input-independent. 
This is restrictive in many real world applications which are characterized by nonstationary and input-dependent noise. This input-dependent nature of the noise significantly deteriorates the control performance, and therefore should be taken into consideration when deriving the optimal control law. Here we mention a few examples from the literature. The first one is the Keck astronomical telescope where the direction of the force of gravity on the mirror changes as the telescope turns to track a star [1]. A second example is the aircraft autolander where the wind disturbance affecting the landing of an aircraft is a function of the altitude of the aircraft [15]. In biomedical applications also different samples have different values of uncertainty depending on whether we have complete or incomplete measurement of that sample. As a final example we mention here communication systems where the systems' noise is nonstationary and data-dependent [4].

Even if the control system has stationary noise affecting its output, the hyperparameter of the tracking error would still be assumed to be input-dependent, especially in on line estimation and control. This can be motivated by considering the sensitivity of the model predictions to noise on the inputs. This leads to a consideration of a model regularizer in which there is a different regularization parameter for different regions in the input space.

In this paper, it will be shown that the assumption of an input independent hyperparameter of tracking error is inconsistent with certain properties of local model approximations. Hence, the method developed in [7] will be extended here to the more general case where the hyperparameter of the tracking error distribution is assumed to be input-dependent. The input-dependency of the hyperparameter implies that it should contribute to the derivation of the optimal control law. This assumption is realistic especially in situations where the noise is nonstationary and where the system is affected by internal (structure), uncertainty and unpredictable dynamics. A Bayesian approach [7] to the calculation of the optimal control law will be taken. It will be shown that incorporating uncertainty of the tracking error when deriving the optimal control law improves not only the transient response of the system but also the steady state response.

This new framework provides an alternative to the standard adaptive control theory and is based on probabilistic control methods rather than deterministic methods. The Bayesian formalism of this paper allows considering model uncertainty as well as parameters uncertainty in the control algorithm. It also extends the method in [7] by allowing the dependency of the hyperparameter of the tracking error on 
the input values. The proposed method provides a natural way to deal with processes characterized by functional uncertainty and nonstationary noise, which are actual conditions under which almost all real world control problems operate as discussed earlier in this section. It takes model uncertainty into consideration when designing the near-to-optimal control law. Taking knowledge of uncertainty into consideration when deriving the near-to-optimal control gives superior control results $[2,5,6]$.

\section{Preliminaries}

In this section, we provide basic elements required for the development of the proposed Bayesian formalism with input-dependent hyperparameter and we discuss the problem formulation.

\subsection{Basic Elements}

Consider the general form of stochastic nonlinear plant given by

$$
y(k+\tau)=f(\mathbf{z}(k), \mathbf{u}(k))+\tilde{\eta}(k+\tau)
$$

which can generally be expressed as

$$
y(k+\tau)=s(\mathbf{z}(k), \mathbf{u}(k), \tilde{\eta}(k+\tau)),
$$

where $y(k+\tau)$ is the system output, $\mathbf{u}(k)$ is the control input, $\tilde{\eta}(k+\tau)$ is an independent noise signal with zero mean Gaussian distribution of variance $\tilde{\rho}, \mathbf{z}(k)=[y(k), \ldots, y(k-q+1), \mathbf{u}(k-1), \ldots, \mathbf{u}(k-$ $p+1)$ ] is the input state vector, $q$ and $p$ are the maximum delays of the output and the input respectively, and $f($.$) and s($.$) are unknown nonlinear functions that represent the system dynamics. Without loss of$ generality it is assumed that $f($.$) and s($.$) are bounded and invertible with respect to \mathbf{u}(k)$.

The aim of control is to design a randomized controller such that the output tracking error is made small and goes to zero, $e_{t}(k+\tau) \longrightarrow 0$,

$$
\begin{aligned}
e_{t}(k+\tau) & =y(k+\tau)-y_{m}(k+\tau) \\
& =s(\mathbf{z}(k), \mathbf{u}(k), \tilde{\eta}(k+\tau))-y_{m}(k+\tau) .
\end{aligned}
$$

This can generally be expressed as

$$
e_{t}(k+\tau)=g\left(y(k+\tau), y_{m}(k+\tau)\right),
$$


where $\mathrm{g}($.$) is the stochastic model of the tracking error and is obtained by subtracting the desired output$ $y_{m}(k+\tau)$ from the system stochastic function $s(\mathbf{z}(k), \mathbf{u}(k), \tilde{\eta}(k+\tau))$.

To achieve the above purpose, design a randomized controller that makes the output tracking error small and goes to zero, we adopt the formalism in [7] which is based on defining the following posterior distribution of control signals, $p\left(\mathbf{u}(k) \mid e_{t}(k+\tau), \mathbf{I}(k)\right)$ using Bayes' rule:

$$
\begin{aligned}
p\left(\mathbf{u}(k) \mid e_{t}(k+\tau), \mathbf{I}(k)\right) & =\frac{p\left(e_{t}(k+\tau) \mid \mathbf{u}(k), \mathbf{I}(k)\right) p(\mathbf{u}(k) \mid \mathbf{I}(k))}{p\left(e_{t}(k+\tau) \mid \mathbf{I}(k)\right)} \\
& =\frac{1}{Z_{S}} \exp \left(-\beta E_{e_{t}}-\alpha E_{u}\right) \\
& =\frac{1}{Z_{S}} \exp (-S(\mathbf{u}(k)))
\end{aligned}
$$

where

$$
S(\mathbf{u}(k))=\beta E_{e_{t}}+\alpha E_{u}
$$

and where $Z_{S}$ is a normalizing constant given by the integral of the numerator over the control input, $\mathbf{u}(k)$. Here, $\mathbf{I}(k)=\{y(1), \ldots, y(k), \mathbf{u}(1), \ldots, \mathbf{u}(k-1)\}$ is the information state vector

The first term $E_{e_{t}}$ is the contribution from the likelihood $p\left(e_{t}(k+\tau) \mid \mathbf{u}(k), \mathbf{I}(k)\right)$ which is assumed to be Gaussian with zero mean and inverse variance $\beta$ :

$$
\begin{aligned}
p\left(e_{t}(k+\tau) \mid \mathbf{u}(k), \mathbf{I}(k)\right) & =\frac{1}{Z_{e_{t}}(\beta)} \exp \left(-\frac{\beta}{2} E_{e_{t}}\right) \\
& =\frac{1}{Z_{e_{t}}(\beta)} \exp \left(-\frac{\beta}{2}\left\{y(k+\tau)-y_{m}(k+\tau)\right\}^{2}\right)
\end{aligned}
$$

where

$$
Z_{e_{t}}(\beta)=\left(\frac{2 \pi}{\beta}\right)^{\frac{1}{2}},
$$

is a normalization factor given by the integral of $E_{e_{t}}$ over the random variable $e_{t}(k+\tau)$, and where $\beta$ is the inverse variance of the distribution of the tracking error. This means that in order to proceed with this control problem, the probability density function (pdf) of the tracking error, $p\left(e_{t}(k+\tau) \mid \mathbf{u}(k), \mathbf{I}(k)\right)$ should be estimated. The pdf of the tracking error will be estimated from process data using recent development in neural networks. It will be discussed briefly in the next section.

The second term $E_{\mathfrak{u}}$ is the contribution from the prior over control signals, which is assumed to be 
Gaussian with zero mean and inverse variance $\alpha$.

$$
\begin{aligned}
p(\mathbf{u}(k) \mid \mathbf{I}(k)) & =\frac{1}{Z_{\mathfrak{u}}(\alpha)} \exp \left(-\alpha E_{\mathfrak{u}}\right) \\
& =\frac{1}{Z_{\mathfrak{u}}(\alpha)} \exp \left(-\frac{\alpha}{2} \mathbf{u}^{\top}(k) \mathbf{u}(k)\right)
\end{aligned}
$$

where $Z_{u}(\alpha)$ is a normalization factor given by

$$
\begin{aligned}
Z_{u}(\alpha) & =\int \exp \left(-\alpha E_{u}\right) d \mathbf{u}, \\
& =\left(\frac{2 \pi}{\alpha}\right)^{\frac{r}{2}},
\end{aligned}
$$

which ensures that $\int p(\mathbf{u}) d \mathbf{u}=1$. Here $r$ is the dimensionality of the control signal.

Remark1: The Gaussian assumption on the prior reflects preference in minimizing the control energy. This corresponds to the penalty term for $\mathbf{u}(\mathrm{k})$, which is usually added in control problems to penalize large control signals [3], reflecting that in practice, the control amplitude needs to be constrained. However, our framework does not insist on smoothness of the control signal and other regularizers can be used.

\subsection{Estimation of the pdf of the Tracking Error}

In order to proceed with the above Bayesian formalism, the unknown distribution of the tracking error should be estimated. Using the probability theory, the density of the tracking error can be obtained from the density of the system output, $y(k+\tau)$ as follows

$$
P_{e_{t}}\left(y(k+\tau), y_{m}(k+\tau)\right)=P_{y}\left(g^{-1}\left(y(k+\tau), y_{m}(k+\tau)\right)\right)\left|\frac{d\left(g^{-1}\left(y(k+\tau), y_{m}(k+\tau)\right)\right)}{d y(k+\tau)}\right|,
$$

where $g^{-1}($.$) is the inverse function of g($.$) with respect to y(k+\tau)$.

Since the transformation (4) is linear, the formula (11) simplifies to

$$
P_{e_{t}}\left(y(k+\tau), y_{m}(k+\tau)\right)=P_{y}\left(e_{t}(k+\tau)+y_{m}(k+\tau)\right) .
$$

The conditional distribution of the system output can be estimated by constructing the following stochastic model [7] for the general nonlinear discrete time system defined in Equation 1:

$$
\mathrm{y}(\mathrm{k}+\tau)=\hat{\mathrm{y}}(\mathrm{k}+\tau)+\eta(k+\tau)
$$


where

$$
\hat{y}(k+\tau)=N_{f}(\mathbf{z}(k), \mathbf{u}(k))
$$

is the prediction of the conditional expectation of the system output produced by a neural network model $N_{f}($.$) . The parameters of the neural network model are to be adjusted using an appropriate$ gradient-based method to optimize a performance function based on the error between the plant and the neural model output. The term $\eta(k+\tau)$ represents uncertainty of the output model, assumed to have Gaussian distribution of zero mean and an input dependent variance, $\rho$. Once the expected value of the system output is estimated, an estimate for the input-dependent variance can be obtained using another neural network, referred to as the variance network, with the same structure and same inputs as that of the output model [7]. To guarantee the positivity of the predicted values of the variance, the $\log$ value of the actual measured variance, $E\left(\|y(k+\tau)-\hat{y}(k+\tau)\|^{2}\right)$, is taken to be the target for the variance network. Consequently, the estimated variance is the exponential of the corresponding output of the variance network:

$$
\hat{\rho}=\exp \{h(\mathbf{z}(k), \mathbf{u}(k))\}
$$

Remark2: The stochastic model (13) does not require the system outputs to have a Gaussian distribution. This however means, that the optimal outputs of the nonlinear approximator (the neural network model optimized using a sum-of-square error) is the input-dependent mean of the Gaussian distribution.

\subsection{Problem Formulation}

Under the Bayesian framework discussed in the previous section, the optimal control law of a stochastic system is derived in [7] by minimizing the expected value of the negative logarithm of the posterior distribution defined in (5) with respect to the control input. Since the normalization factor, $Z_{S}$ is evaluated by integrating over the control signal, the minimization process is shown [7] to be equivalent to minimizing $\underset{\eta(k+\tau)}{E}[S(\mathbf{u}(k))]$ given by Equation (6). For the prior distribution given in Equation (9) and noise model given by Equation (7) this can be written in the form

$$
\underset{\eta(k+\tau)}{E}[S(\mathbf{u}(k))]=\underset{\eta(k+\tau)}{E}\left[\frac{\beta}{2}\left\{\mathrm{y}(\mathrm{k}+\tau)-\mathrm{y}_{\mathrm{m}}(\mathrm{k}+\tau)\right\}^{2}+\frac{\alpha}{2} \mathbf{u}^{\top}(\mathrm{k}) \mathbf{u}(\mathrm{k})\right]
$$


Using Equation (13) in Equation (16) yields

$$
\begin{array}{r}
\underset{\eta(k+\tau)}{E}[S(\mathbf{u}(k))]=\underset{\eta(k+\tau)}{E}\left[\frac{\beta}{2}\left\{\hat{y}(k+\tau)+\eta(k+\tau)-y_{m}(k+\tau)\right\}^{2}+\frac{\alpha}{2} \mathbf{u}^{\top}(k) \mathbf{u}(k)\right] \\
=\frac{\beta}{2}\left[\left\{\hat{y}(k+\tau)-y_{m}(k+\tau)\right\}^{2}+2\left\{\hat{y}(k+\tau)-y_{m}(k+\tau)\right\} E[\eta(k+\tau)]\right. \\
+E\left[\eta^{2}(k+\tau)\right]+\frac{\alpha}{2} \mathbf{u}^{\top}(k) \mathbf{u}(k) .
\end{array}
$$

Using the facts that $E[\eta(\mathrm{k}+\tau)]=0$ and $E\left[\eta^{2}(\mathrm{k}+\tau)\right]=\rho$ yields

$$
\underset{\eta(k+\tau)}{E}[S(\mathbf{u}(k))]=\frac{\beta}{2}\left[\left\{\hat{y}(k+\tau)-y_{m}(k+\tau)\right\}^{2}+\rho\right]+\frac{\alpha}{2} \mathbf{u}^{\top}(k) \mathbf{u}(k) .
$$

The performance index (18) is then minimized with respect to the control input and the optimal control law is derived [7]. The derivation of the optimal control law in [7] is based on the assumption of an input independent hyperparameter of the tracking error, given by the inverse variance $\beta$ of the tracking error distribution. However, assuming that the hyperparameter of the tracking error is input independent is inconsistent with certain properties of local model approximations. To illustrate the problem of local model approximation we demonstrate on minimizing a sum of square error between the desired output and the system output. In particular, we will show that this mean square tracking error will have zero expected value and an input dependent variance which can be estimated using another neural network.

Consider the output of a network which performs a mapping from input variables $\mathbf{x}(\mathrm{k})=$ $\{\mathbf{z}(k), \mathbf{u}(k)\}$ to an output variable $y(k+\tau)$. The mean square tracking error is then given by an integral of the form,

$$
E_{e_{t}}=\frac{1}{2} \int\left\{y_{m}(k+\tau)-y(k+\tau)\right\}^{2} d y(k+\tau)
$$

Following the discussion in Section 2.2, the tracking error distribution can be obtained from the distribution of the system output. For that purpose we define the following conditional averages of the system output $y(k+\tau)$

$$
\begin{aligned}
\langle y(k+\tau)| \mathbf{x}(k)> & =\int y(k+\tau) p(y(k+\tau) \mid \mathbf{x}(k)) d y(k+\tau), \\
\left\langle y^{2}(k+\tau)\right| \mathbf{x}(k)> & =\int y^{2}(k+\tau) p(y(k+\tau) \mid \mathbf{x}(k)) d y(k+\tau) .
\end{aligned}
$$


Rewrite the term in brackets in Equation (19) in the form

$$
\begin{gathered}
\left\{\mathrm{y}_{\mathrm{m}}(\mathrm{k}+\tau)-\mathrm{y}(\mathrm{k}+\tau)\right\}^{2}=\left\{\mathrm{y}_{\mathrm{m}}(\mathrm{k}+\tau)-<\mathrm{y}(\mathrm{k}+\tau)|\mathbf{x}(\mathrm{k})>+<\mathrm{y}(\mathrm{k}+\tau)| \mathbf{x}(\mathrm{k})>-\mathrm{y}(\mathrm{k}+\tau)\right\}^{2} \\
=\left\{\mathrm{y}_{\mathrm{m}}(\mathrm{k}+\tau)-<\mathrm{y}(\mathrm{k}+\tau) \mid \mathbf{x}(\mathrm{k})>\right\}^{2}+\{<\mathrm{y}(\mathrm{k}+\tau) \mid \mathbf{x}(\mathrm{k})>-\mathrm{y}(\mathrm{k}+\tau)\}^{2} \\
\left.\quad+2\left\{\mathrm{y}_{\mathrm{m}}(\mathrm{k}+\tau)-<\mathrm{y}(\mathrm{k}+\tau) \mid \mathbf{x}(\mathrm{k})>\right\}\langle\mathrm{y}(\mathrm{k}+\tau)| \mathbf{x}(\mathrm{k})>-\mathrm{y}(\mathrm{k}+\tau)\right\}, \quad(22)
\end{gathered}
$$

Next substitute Equation (22) into Equation (19) and make use of Equations (20) and (21), the third term on the right hand side of (22) then vanishes as a consequence of the integration over $y(k+\tau)$. This yields

$$
E_{e_{t}}=\frac{1}{2}\left\{y_{m}(k+\tau)-<y(k+\tau) \mid \mathbf{x}(k)>\right\}^{2}+\frac{1}{2}\left\{<y^{2}(k+\tau)|\mathbf{x}(k)>-<y(k+\tau)| \mathbf{x}(k)>^{2}\right\} .
$$

The first term in this equation is the bias. It measures the extent to which the expected value of the system output differs from the desired output value. The second term is the variance which measures the extent to which the expected output value is sensitive to a particular choice of control input. Equation (23) shows that the expressions for Bias and variance are functions of the control signal $\mathbf{u}(k)$. Therefore, to achieve good overall performance the bias and the variance of the square of the tracking error which are input dependent would both have to be minimized. Thus for local model approximation and control where the bias and variance of the tracking error are input dependent, it is not sufficient to assume that the probability model of the tracking error has an input independent variance. To be consistent with our prior knowledge it is necessary to make the variance of the probability model of the tracking error depends on the input vector. This contrasts with the input independent hyperparameter proposed in [7], where the tracking error uncertainty does not alter the optimal control law. In the next section we extend the results in [7] by allowing the dependency of the hyperparameter of the tracking error on the input variables.

\section{Solution Development and Analysis: Main Results}

\subsection{Derivation of the Optimal Control Law}

For analyzing the Bayesian control problem with an input dependent hyperparameter of the tracking error, it is assumed that although the tracking error is unknown, a model for estimating its variance is 
available. To do so, a nonlinear neural network can be used to provide an estimate for the variance of the tracking error as follows:

$$
\sigma_{e_{t}}^{2}=\exp \left\{N_{\sigma}(\mathbf{z}(k), \mathbf{u}(k))\right\}
$$

Using Equations (24) and (15) in Equation (18) yields the following performance index to be minimized:

$$
\underset{\eta(k+\tau)}{E}[S(\mathbf{u}(k))]=\frac{1}{2 \sigma_{e_{t}}^{2}}\left[\left\{\hat{y}(k+\tau)-y_{m}(k+\tau)\right\}^{2}+\exp \{h(\mathbf{z}(k), \mathbf{u}(k))\}\right]+\frac{\alpha}{2} \mathbf{u}^{\top}(k) \mathbf{u}(k) .
$$

Minimization of the explicit performance index (25) leads to the optimality equation specified in the following theorem.

Theorem 1. The optimal control law minimizing the expectation of the negative logarithm of the posterior of Equation (25) subject to the system of Equation (14) and variances of system and tracking error of Equations (15) and (24) respectively can be found by solving the following nonlinear optimality equation:

$$
\begin{array}{r}
0=-2 \frac{\partial \sigma_{e_{t}}^{2}}{\partial \mathbf{u}(k)} \frac{\left[\hat{y}(k+\tau)-y_{m}(k+\tau)\right]^{2}}{\left[2 \sigma_{e_{t}}^{2}\right]^{2}}+\frac{\left[\hat{y}(k+\tau)-y_{m}(k+\tau)\right]}{\sigma_{e_{t}}^{2}} \frac{\partial \hat{y}(k+\tau)}{\partial \mathbf{u}(k)} \\
-2 \frac{\partial \sigma_{e_{t}}^{2}}{\partial \mathbf{u}(k)} \frac{\hat{\rho}^{2}}{\left[2 \sigma_{e_{t}}^{2}\right]^{2}}+\frac{1}{2 \sigma_{e_{t}}^{2}} \frac{\partial \hat{\rho}^{2}}{\partial \mathbf{u}(k)}+\alpha \mathbf{u}(k) .
\end{array}
$$

where this equation is obtained by setting the derivative of Equation (25) with respect to the control signal equal to zero.

Remark3: Since the forward model of the system output and the model of its variance and the model of the hyperparameter of the tracking error are nonlinear functions of the control signal, a nonlinear optimization method is required for solving Equation (26) and deriving the optimal control law which is then used to update the parameters of the neural network controller. As such the optimization algorithm (26) can only guarantee the search for local minima.

\subsection{Predictive Distribution of the System Output}

The Bayesian formalism above is used for estimating the posterior distribution of control signals. According to this formulation, the distribution of control signals affects the distribution of the system output and in-turn, the distribution of the tracking error. In addition, there will be a contribution of the tracking error distribution arising from the assumed Gaussian noise on the error tracking. 
Using the rules of probability, the predictive distribution of the system output for a given input can be written in the form

$$
\begin{aligned}
& p\left(\eta(k+\tau) \mid e_{t}(k+\tau), \mathbf{z}(k), \mathbf{I}(k)\right)= \\
& \quad \int p(\eta(k+\tau) \mid \mathbf{u}(k), \mathbf{z}(k), \mathbf{I}(k)) p\left(\mathbf{u}(k) \mid e_{t}(k+\tau), \mathbf{I}(k)\right) d \mathbf{u}(k),
\end{aligned}
$$

where $p\left(\boldsymbol{u}(k) \mid e_{t}(k+\tau), \mathbf{I}(k)\right)$ is the posterior distribution of the control signal. The distribution $p(\eta(k+\tau) \mid \mathbf{u}(k), \mathbf{z}(k), \mathbf{I}(k))$ is the distribution of the noise on the system output, which can be obtained by substituting Equation (13) into Equation (7) as follows

$$
p(\eta(k+\tau) \mid \mathbf{u}(k), \mathbf{z}(k), \mathbf{I}(k))=\frac{1}{Z_{e_{t}}(\beta)} \exp \left(-\frac{\beta}{2}\left\{\hat{y}(k+\tau)+\eta(k+\tau)-y_{m}(k+\tau)\right\}^{2}\right) .
$$

The integral in Equation (27) is not analytically tractable for nonlinear models. In the Bayesian work for estimating the weight parameters of neural networks, Mackay [12] used a Gaussian approximation for the posterior distribution of the weight vector to solve this problem. Similarly, here we use the Gaussian approximation for the posterior distribution of the control signal. This is obtained by considering the Taylor expansion of $E[\mathrm{~S}(\mathbf{u})]$ around its minimum value of $\mathbf{u}_{\mathrm{MP}}(\mathrm{k})$ and retaining terms up to the second order so that

$$
E[S(\mathbf{u}(k))]=E\left[S\left(\mathbf{u}_{M P}(k)\right)\right]+\frac{1}{2}\left(\mathbf{u}(k)-\mathbf{u}_{M P}(k)\right) A\left(\mathbf{u}(k)-\mathbf{u}_{M P}(k)\right)
$$

where the linear term has vanished since we are expanding around a minimum of $E[S(\mathbf{u})]$. Here $A$ is the Hessian matrix of the total error function, with elements given by

$$
\begin{aligned}
A & =\frac{-\nabla^{2} \sigma_{e_{t}}^{2}\left[\sigma_{e_{t}}^{2}\right]^{2}+2 \sigma_{e_{t}}^{2}\left[\nabla \sigma_{e_{t}}^{2}\right]^{2}}{\left[\sigma_{e_{t}}^{2}\right]^{4}} E_{\hat{e}_{t}}-\frac{2 \nabla E_{\hat{e}_{t}} \nabla \sigma_{e_{t}}^{2}}{\left[\sigma_{e_{t}}^{2}\right]^{2}} \\
& +\frac{1}{\sigma_{e_{t}}^{2}} \nabla^{2} E_{\hat{e_{t}}}+\frac{\nabla^{2} \hat{\rho}^{2}}{2 \sigma_{e_{t}}^{2}}-\frac{\nabla \sigma_{e_{t}}^{2} \nabla \hat{\rho}^{2}}{\left[\sigma_{e_{t}}^{2}\right]^{2}}-\frac{\nabla^{2} \sigma_{e_{t}}^{2} \hat{\rho}^{2}}{2\left[\sigma_{e_{t}}^{2}\right]^{2}}+\frac{\left(\nabla \sigma_{e_{t}}^{2}\right)^{2} \hat{\rho}^{2}}{\left[\sigma_{e_{t}}^{2}\right]^{3}}+\alpha \nabla^{2} E_{u} \\
& =-\beta^{2}\left(\nabla^{2} \sigma_{e_{t}}^{2} E_{\hat{e}_{t}}+\frac{\nabla^{2} \sigma_{e_{t}}^{2} \hat{\rho}^{2}}{2}\right)+\beta^{3}\left(2\left[\nabla \sigma_{e_{t}}^{2}\right]^{2} E_{\hat{e_{t}}}+\left[\nabla \sigma_{e_{t}}^{2}\right]^{2} \hat{\rho}^{2}\right) \\
& -\beta^{2}\left(2 \nabla E_{\hat{e_{t}}} \nabla \sigma_{e_{t}}^{2}+\nabla \sigma_{e_{t}}^{2} \nabla \hat{\rho}^{2}\right)+\beta\left(\nabla^{2} E_{\hat{e_{t}}}+\frac{\nabla^{2} \hat{\rho}^{2}}{2}\right)+\alpha I, \\
& =-\beta^{2}\left(\nabla^{2} \sigma_{e_{t}}^{2} E_{\hat{e_{t}}}+\frac{\nabla^{2} \sigma_{e_{t}}^{2} \hat{\rho}^{2}}{2}+2 \nabla E_{\hat{e_{t}}} \nabla \sigma_{e_{t}}^{2}+\nabla \sigma_{e_{t}}^{2} \nabla \hat{\rho}^{2}\right) \\
& +\beta^{3}\left(2\left[\nabla \sigma_{e_{t}}^{2}\right]^{2} E_{\hat{e_{t}}}+\left[\nabla \sigma_{e_{t}}^{2}\right]^{2} \hat{\rho}^{2}\right)+\beta\left(\nabla^{2} E_{\hat{e}_{t}}+\frac{\nabla^{2} \hat{\rho}^{2}}{2}\right)+\alpha I,
\end{aligned}
$$


where $E_{\hat{e_{t}}}=(1 / 2)\left\{\hat{y}(k+\tau)-y_{m}(k+\tau)\right\}^{2}$. Note that all terms in Equation (30) are evaluated at the most probable control value, $\mathbf{u}_{\mathrm{MP}}(\mathrm{k})$.

Using the Laplace approximation given in (29) and the model of the variance of the tracking error given in Equation (24), the predictive distribution of the system output as defined in Equation (27) can be written as

$$
\begin{array}{r}
p\left(\eta(k+\tau) \mid e_{t}(k+\tau), \mathbf{z}(k), \mathbf{I}(k)\right) \\
\propto \int \exp \left(-\frac{1}{2 \sigma_{e_{t}}^{2}}\left\{\eta(k+\tau)+\hat{y}(k+\tau)-y_{m}(k+\tau)\right\}^{2}\right) \\
\quad \exp \left(-\frac{1}{2} \triangle \mathbf{u}^{\top}(k) A \triangle \mathbf{u}(k)\right) d \triangle \mathbf{u}(k),
\end{array}
$$

where $\triangle \mathbf{u}(k)=\mathbf{u}(k)-\mathbf{u}_{M P}(k)$. Assuming that the width of the posterior distribution of control signals (determined by the Hessian matrix $A$ ) is sufficiently narrow, the nonlinear functions $\hat{y}(k+\tau)=$ $N_{f}(\mathbf{z}(k), \mathbf{u}(k))$ and $\sigma_{e_{t}}^{2}$ may now be approximated by their Taylor expansions about $\mathbf{u}_{M P}(k)$ as follows

$$
\begin{aligned}
\hat{y}(k+\tau) & =\hat{y}_{M P}(k+\tau)+J^{\top} \triangle \mathbf{u}(k) \\
\sigma_{e_{t}}^{2} & =\sigma_{e_{t}}^{2^{M P}}+v^{\top} \triangle \mathbf{u}(k)+\frac{1}{2} \triangle \mathbf{u}^{\top}(k) M \triangle \mathbf{u}(k)
\end{aligned}
$$

where

$$
\begin{aligned}
J & \left.\equiv \nabla_{\mathbf{u}(k)} \hat{y}(k+\tau)\right|_{\mathbf{u}_{M P}(k)} \\
v & \left.\equiv \nabla_{\mathbf{u}} \sigma_{e_{t}}^{2}\right|_{\mathbf{u}_{M P}(k)} \\
M & \left.\equiv \nabla_{\mathbf{u}}^{2} \sigma_{e_{t}}^{2}\right|_{\mathbf{u}_{M P}(k)} .
\end{aligned}
$$

Using Equation (32) in Equation (31) yields

$$
\begin{aligned}
& p\left(\eta(k+\tau) \mid e_{t}(k+\tau), \mathbf{z}(k), \mathbf{I}(k)\right) \propto \\
& \int \exp \left(-\frac{1}{2\left\{\sigma_{e_{t}}^{2^{M P}}+v^{\top} \triangle \mathbf{u}(k)+\frac{1}{2} \triangle \mathbf{u}^{\top}(k) M \triangle \mathbf{u}(k)\right\}}\left\{\hat{y}_{M P}(k+\tau)+J^{\top} \triangle \mathbf{u}(k)+\eta(k+\tau)-y_{m}(k+\tau)\right\}^{2}\right) \\
& \exp \left(-\frac{1}{2} \triangle \mathbf{u}^{\top}(\mathrm{k}) A \triangle \mathbf{u}(\mathrm{k})\right) \mathrm{d} \triangle \mathbf{u}(\mathrm{k})
\end{aligned}
$$

where $\hat{y}_{M P}(k+\tau)=\left.\hat{y}(k+\tau)\right|_{\mathbf{u}_{M P}}$. The integral in Equation (34) can be evaluated as given in the 
Appendix to give a Gaussian distribution of the form

$p\left(\eta(k+\tau) \mid e_{t}(k+\tau), \mathbf{z}(k), \mathbf{I}(k)\right)=\frac{1}{\left(2 \pi \sigma_{\eta}^{2}\right)^{1 / 2}} \exp \left(-\frac{\left(\eta(k+\tau)-\left[y_{m}(k+\tau)-\hat{y}_{M P}(k+\tau)\right]\right)^{2}}{2 \sigma_{\eta}^{2}}\right)$.

This distribution has a mean given by $y_{m}(k+\tau)-\hat{y}_{M P}(k+\tau)$ and a variance given by

$$
\sigma_{\eta}^{2}=\sigma_{e_{t}}^{2^{M P}}+J^{\top} A^{-1} J+\frac{1}{2} \operatorname{Tr}\left(A^{-1} M\right)
$$

However, since the mean value of the residual error of the system output is zero [7] it follows that $\hat{y}_{M P}(k+\tau)=y_{m}(k+\tau)$. This means that after training the forward and inverse models, the expected value of the system output will be equal to the desired output value.

It is clear from Equation (36) that the standard deviation of the predictive distribution of the system output has three contributions. The first component is the variance of intrinsic noise on the tracking error but is now dependent on the control input. The second component arises from the width of the posterior distribution of control signals around the system output. And the third component arises from the width of the posterior distribution of control signals around the noise variance of tracking error.

\subsection{Estimation of the Hyperparameters, $\alpha$ and $\beta$}

For the input-dependent hyperparameter of the tracking error, the values of the hyperparameters which maximizes the posterior distribution of control signal can be found by implementing the same procedure used in [7]. It is based on maximizing the likelihood function, $p\left(e_{t}(k+\tau) \mid \alpha, \beta\right)$ which is called the evidence for $\alpha$ and $\beta$,

$$
\begin{aligned}
p\left(e_{t}(k+\tau) \mid \alpha, \beta\right) & =\int p\left(e_{t}(k+\tau) \mid \mathbf{u}(k), \alpha, \beta\right) p(\mathbf{u}(k) \mid \alpha, \beta) d \mathbf{u}(k), \\
& =\int p\left(e_{t}(k+\tau) \mid \mathbf{u}(k), \beta\right) p(\mathbf{u}(k) \mid \alpha) d \mathbf{u}(k),
\end{aligned}
$$

where it is made use of the fact that the prior is independent of $\beta$ and the likelihood function is independent of $\alpha$. Using the exponential forms given in Equations (9) and (7) for the prior distribution of control signals and the likelihood distribution, together with Equation (6), the evidence of $\alpha$ and $\beta$ can then be written in the form

$$
\begin{aligned}
p\left(e_{\mathrm{t}}(k+\tau) \mid \alpha, \beta\right) & =\frac{1}{Z_{e_{\mathrm{t}}}(\beta)} \frac{1}{Z_{\mathfrak{u}}(\alpha)} \int \exp -S(\mathbf{u}(k)) d \mathbf{u}(k) \\
& =\frac{Z_{S}(\alpha, \beta)}{Z_{e_{\mathrm{t}}}(\beta) Z_{\mathfrak{u}}(\alpha)} .
\end{aligned}
$$


The normalization coefficients $Z_{e_{t}}(\beta)$ and $Z_{u}(\alpha)$ have already been evaluated in Equations (8) and (10) respectively. For the Gaussian approximation of the posterior distribution of control signals, $Z_{S}(\alpha, \beta)$ is given by,

$$
Z_{S}^{*}(\alpha, \beta)=e^{-E\left[S\left(\mathbf{u}_{\mathcal{M P}}(k)\right)\right]}(2 \pi)^{r / 2}|A|^{-\frac{1}{2}},
$$

where $A$ is the Hessian matrix of the error function defined in Equation (30). Using Equations (39), (8) and (10) the $\log$ of the evidence for $\alpha$ and $\beta$ is then given by,

$$
\ln p\left(e_{t}(k+\tau) \mid \alpha, \beta\right)=-\alpha E_{u}^{M P}-\beta E_{E\left[e_{t}\right]}^{M P}-\frac{1}{2} \ln |A|+\frac{r}{2} \ln \alpha+\frac{1}{2} \ln \beta-\frac{1}{2} \ln (2 \pi) .
$$

Maximization of the explicit form of the $\log$ of the evidence defined in Equation (40) subject to Equations (39), (8) and (10) leads to re-estimation equations for $\alpha$ and $\beta$ defined in the following theorem.

Theorem 2. The hyperparameters $\alpha$ and $\beta$ maximizing the log of the evidence defined in Equation (40) subject to Equations (39), (8) and (10) are given by

$$
\begin{aligned}
\alpha^{\text {new }} & =\frac{\gamma}{2 \mathrm{E}_{\mathrm{u}}}, \\
\beta^{\text {new }} & =\frac{1-\gamma}{2 \mathrm{E}_{E\left[e_{\mathrm{t}}\right]}},
\end{aligned}
$$

where the quantity $\gamma$ is defined by

$$
\gamma=\sum_{i=1}^{r} \frac{2 \mu_{i}+3 \lambda_{i}+\vartheta_{i}}{\mu_{i}+\lambda_{i}+\vartheta_{i}+\alpha}
$$

and where $\mu_{i}, \lambda_{i}$, and $\vartheta_{i}$ denote the eigenvalues of the first Hessian matrix, the second Hessian matrix, and the third Hessian matrix represented by the first term, the second term, and the third term respectively of Equation (30) for the general nonlinear class of discrete time systems.

The proof of theorem 2 is given in the Appendix. It can easily be carried out by taking the derivative of Equation (40) with respect to $\alpha$ and $\beta$ and setting the derivative equal to zero.

\section{Affine Class of Nonlinear Discrete Time Systems}

The theory developed in the previous section is applied here to a stochastic multi-input single-output affine class of nonlinear discrete time systems having the general form

$$
y(k+\tau)=f[\mathbf{z}(k)]+\mathbf{g}^{\top}[\mathbf{z}(k)] \mathbf{u}(k)+\tilde{\eta}(k+\tau),
$$


where $y(k+\tau)$ is the output, $\mathbf{u}(k)$ is the control input vector of dimension $r, \mathbf{z}(k)=[y(k), \ldots, y(k-$ $q+1), \mathbf{u}(k-1), \ldots, \mathbf{u}(k-p+1)]$ is the input state vector, $f[\mathbf{z}(k)]: \mathcal{R}^{q+r p-r} \longmapsto \mathcal{R}$ and $\mathbf{g}[\mathbf{z}(\mathrm{k})]: \mathcal{R}^{\mathrm{q}+\mathrm{rp}-\mathrm{r}} \longmapsto \mathcal{R}^{\mathrm{r}}$ are unknown nonlinear functions of the state and $\tilde{\eta}(\mathrm{k}+\tau)$ is an additive noise signal.

Two neural networks can then be used to approximate the nonlinear functions $f[\mathbf{z}(k)]$ and $g[\mathbf{z}(k)]$. The networks' estimates are denoted as $\hat{f}[\mathbf{z}(k)]$ and $\hat{g}[\mathbf{z}(k)]$. Hence, the expected value of the system output is given by

$$
\hat{y}(k+\tau)=\hat{f}[\mathbf{z}(k)]+\hat{\mathbf{g}}^{\top}[\mathbf{z}(k)] \mathbf{u}(k) .
$$

The variance of the tracking error, $e_{t}$ denoted by the inverse hyperparameter, $\frac{1}{\beta}$ and the variance of the residual error of the system output, are estimated using two neural network models as follows:

$$
\begin{aligned}
\frac{1}{\beta} & =\sigma_{e_{t}}^{2}=\mathbf{q}^{\top}[\mathbf{z}(k)] \mathbf{u}(k) . \\
\hat{\rho} & =\mathbf{h}^{\top}[\mathbf{z}(k)] \mathbf{u}(k) .
\end{aligned}
$$

To guarantee the positivity of the predicted variance values, the method of Lagrange multipliers is assumed here to optimize the parameters of the variance network, instead of the exponential transformation in the general class of nonlinear control problems. By using the method of Lagrange multipliers, the linearity of the affine class problems can be preserved. Using Equations (47) and (46) in Equation (18) yields

$$
\underset{\eta(k+\tau)}{E}[S(\mathbf{u}(k))]=\frac{1}{2\left(\mathbf{q}^{\top}[\mathbf{z}(k)] \mathbf{u}(k)\right)}\left[\left\{\hat{y}(k+\tau)-y_{m}(k+\tau)\right\}^{2}+\mathbf{h}^{\top}[\mathbf{z}(k)] \mathbf{u}(k)\right]+\frac{\alpha}{2} \mathbf{u}^{\top}(k) \mathbf{u}(k) .
$$

Setting the derivative of Equation (48) with respect to the control signal equal to zero leads to control law as a solution of the optimality equation specified in the following theorem.

Theorem 3. The optimal control law minimizing the performance index of Equation (48) subject to the system of Equation (45) and variances of tracking errors and system of Equations (46) and (47) respectively can be found by solving the following cubic optimality equation:

$$
\begin{aligned}
0 & =4 \alpha \mathbf{u}^{\top}(k) \mathbf{q}[\mathbf{z}(k)] \mathbf{q}^{\top}[\mathbf{z}(k)] \mathbf{u}(k) \mathbf{u}(k)+4 \mathbf{u}^{\top}(k) \hat{\mathbf{g}}[\mathbf{z}(k)] \mathbf{q}^{\top}[\mathbf{z}(k)] \mathbf{u}(k) \hat{\mathbf{g}}[\mathbf{z}(k)] \\
& -2 \mathbf{u}^{\top}(k) \hat{\mathbf{g}}[\mathbf{z}(k)] \hat{\mathbf{g}}^{\top}[\mathbf{z}(k)] \mathbf{u}(k) \mathbf{q}[\mathbf{z}(k)]+2 \mathbf{q}^{\top}[\mathbf{z}(k)] \mathbf{u}(k) \mathbf{h}[\mathbf{z}(k)]-2 \mathbf{h}^{\top}[\mathbf{z}(k)] \mathbf{u}(k) \mathbf{q}[\mathbf{z}(k)] \\
& +4\left\{\hat{f}[\mathbf{z}(k)]-y_{m}(k+\tau)\right\}\left[\mathbf{q}^{\top}[\mathbf{z}(k)] \mathbf{u}(k) \hat{\mathbf{g}}[\mathbf{z}(k)]-\hat{\mathbf{g}}^{\top}[\mathbf{z}(k)] \mathbf{u}(k) \mathbf{q}[\mathbf{z}(k)]\right] \\
& -2 \mathbf{q}[\mathbf{z}(k)]\left\{\hat{\mathbf{f}}[\mathbf{z}(k)]-y_{m}(k+\tau)\right\}^{2}
\end{aligned}
$$


For the single-input single-output discrete time affine nonlinear systems, the above equation reduces to

$$
0=4 \alpha u^{3}(k) q^{2}[\mathbf{z}(k)]+2 u^{2}(k) \widehat{g}^{2}[\mathbf{z}(k)] \mathbf{q}[\mathbf{z}(k)]-2 q[\mathbf{z}(k)]\left\{\hat{f}[\mathbf{z}(k)]-y_{m}(k+\tau)\right\}^{2}
$$

This cubic equation could be solved numerically or analytically in closed form. Depending on the sign of the discriminant, the solution of a cubic equation could have 1 real root, 3 real roots with two or three equal roots or 3 distinct real roots [18]. Because, the sign of the discriminant of Equation (50) is not determined, any of the above solutions could be valid. This implies that a decision should be taken here to determine the control signal to be forwarded to the plant. For the case of three real solutions one of them is a local maximum. In order to exclude the maximum from the set of possible solutions, the second derivative of the error equation could be evaluated. One of the possibilities to choose the control signal to be forwarded to the plant amongst the remaining solutions is to take the control signal which gives the minimum value of the error function defined by Equation (48). This corresponds to maximizing the likelihood function. To treat the problem more properly in a Bayesian framework, another alternative for choosing the control signal to be forwarded to the plant is to integrate over all possible control signals. This corresponds to the maximum aposteriori estimate.

For the multi-input single-output nonlinear affine discrete time systems the optimal control signal needs to be obtained by solving the full vector cubic equation (49) numerically.

Using the neural estimates of the nonlinear models of Equation (45) together with the neural network estimates for the variance of the tracking error defined in Equation (46), in Equation (31) yields the distribution for $\eta(k+\tau)$ defined by the following integral:

$$
\begin{aligned}
& p\left(\eta(k+\tau) \mid e_{t}(k+\tau), \mathbf{z}(k), \mathbf{I}(k)\right) \propto \\
& \left.\int \exp \left(-\frac{1}{2\left\{\mathbf{q}^{\top}[\mathbf{z}(\mathrm{k})] \mathbf{u}(\mathrm{k})\right\}}\{\hat{\mathrm{f}} \mathbf{z}(\mathrm{k})]+\hat{\mathbf{g}}^{\mathrm{T}}[\mathbf{z}(\mathrm{k})] \mathbf{u}(\mathrm{k})+\eta(\mathrm{k}+\tau)-\mathrm{y}_{\mathbf{m}}(\mathrm{k}+\tau)\right\}^{2}\right) \\
& \exp \left(-\frac{1}{2} \triangle \mathbf{u}^{\mathrm{T}}(\mathrm{k}) \mathrm{A} \triangle \mathbf{u}(\mathrm{k})\right) \mathrm{d} \triangle \mathbf{u}(\mathrm{k}) \\
& \left.=\int \exp \left(-\frac{1}{2\left\{\mathbf{q}^{\top}[\mathbf{z}(k)]\left[\mathbf{u}_{M P}(k)+\triangle \mathbf{u}(k)\right]\right\}}\{\hat{f} \hat{\mathbf{z}}(\mathrm{k})]+\hat{\mathbf{g}}^{\top}[\mathbf{z}(k)]\left[\mathbf{u}_{M P}(k)+\triangle \mathbf{u}(k)\right]+\eta(k+\tau)-y_{\mathfrak{m}}(k+\tau)\right\}^{2}\right) \\
& \exp \left(-\frac{1}{2} \triangle \mathbf{u}^{\top}(k) A \triangle \mathbf{u}(k)\right) \mathrm{d} \triangle \mathbf{u}(k)
\end{aligned}
$$

The evaluation of the integral in Equation (51) is given in the Appendix. It is a Gaussian distribution 
of the form

$$
\begin{aligned}
p\left(\eta(k+\tau) \mid e_{t}(k+\tau), \mathbf{z}(k), \mathbf{I}(k)\right) & \\
= & \frac{1}{\left(2 \pi \sigma_{\eta}^{2}\right)^{1 / 2}} \exp \left(-\frac{\left\{\eta(k+\tau)-\left[y_{m}(k+\tau)-\hat{f}[\mathbf{x}(k)]-\hat{\mathbf{g}}^{\top}[\mathbf{z}(k)] \mathbf{u}_{M P}(k)\right]\right\}^{2}}{2 \sigma_{\eta}^{2}}\right) .
\end{aligned}
$$

This distribution has a mean given by $\mathrm{y}_{\mathrm{m}}(\mathrm{k}+\tau)-\left\{\hat{\mathrm{f}}[\mathbf{z}(\mathrm{k})]+\hat{\mathbf{g}}^{\top}[\mathbf{z}(\mathrm{k})] \mathbf{u}_{M P}(\mathrm{k})\right\}$, and a variance given by

$$
\sigma_{\eta}^{2}=\mathbf{q}^{\top}[\mathbf{z}(k)] \mathbf{u}_{M P}(k)+\hat{\mathbf{g}}^{\top}[\mathbf{z}(k)] A^{-1} \hat{\mathbf{g}}[\mathbf{z}(k)]
$$

where $A$ is the Hessian of the error function of the affine system defined in Equation (48)

$$
\begin{aligned}
A & =\frac{\nabla^{2} E_{\hat{e_{t}}}}{2 \sigma_{e_{t}}^{2}}-\frac{4 \nabla \sigma_{e_{t}}^{2} \nabla E_{\hat{e_{t}}}}{\left[2 \sigma_{e_{t}}^{2}\right]^{2}}+\frac{8\left(\nabla \sigma_{e_{t}}^{2}\right)^{2} E_{\hat{e_{t}}}}{\left[2 \sigma_{e_{t}}^{2}\right]^{3}}-\frac{4 \nabla \sigma_{e_{t}}^{2} \mathbf{h}}{\left[2 \sigma_{e_{t}}^{2}\right]^{2}}+\frac{8\left(\nabla \sigma_{e_{t}}^{2}\right)^{2} \mathbf{h}^{\top} \mathbf{u}}{\left[2 \sigma_{e_{t}}^{2}\right]^{3}}+\alpha I \\
& =\beta \nabla^{2} E_{\hat{e_{t}}}-4 \beta^{2} \nabla \sigma_{e_{t}}^{2} \nabla E_{\hat{e_{t}}}+8 \beta^{3}\left(\nabla \sigma_{e_{t}}^{2}\right)^{2} E_{\hat{e_{t}}}-4 \beta^{2} \nabla \sigma_{e_{t}}^{2} \mathbf{h}+8 \beta^{3}\left(\nabla \sigma_{e_{t}}^{2}\right)^{2} \mathbf{h}^{\top} \mathbf{u}+\alpha I, \\
& =-\beta^{2}\left\{4 \nabla \sigma_{e_{t}}^{2} \nabla E_{\hat{e_{t}}}+4 \nabla \sigma_{e_{t}}^{2} \mathbf{h}\right\}+\beta^{3}\left\{8\left(\nabla \sigma_{e_{t}}^{2}\right)^{2} E_{\hat{e_{t}}}+8\left(\nabla \sigma_{e_{t}}^{2}\right)^{2} \mathbf{h}^{\top} \mathbf{u}\right\}+\beta\left\{\nabla^{2} E_{\hat{e_{t}}}\right\}+\alpha I .
\end{aligned}
$$

Again since the mean value of the residual error of the system output is zero, it follows that $\hat{f}[\mathbf{z}(k)]+\hat{\mathbf{g}}^{\top}[\mathbf{z}(k)] \mathbf{u}_{M P}(k)=y_{m}(k+\tau)$. This means that, after training the forward and inverse models the expected value of the system output will be equal to the desired output value.

It is clear from Equation (53) that the standard deviation of the predictive distribution of the residual error of the system output has two contributions. The first contribution arises from intrinsic noise on the tracking error, represented by the first term of Equation (53). The second contribution arises from the width of the posterior distribution of control signals, represented by the second term of Equation (53).

Finally the same update equations given in Theorem 2 for the hyperparameters $\alpha$, and $\beta$ can be shown to be applicable for the affine class of discrete time systems, taking into consideration the Hessian matrix of the affine systems specified in Equation (54).

\section{Simulation Example}

In this section a nonlinear SISO stochastic control problem is simulated. The dynamic equation of the system is

$$
y(k+1)=\sin [y(k)]+\cos [3 y(k)]+\{2+\cos [y(k)]\} u(k)+\epsilon(k+1)
$$


where $\epsilon(k+1)$ was assumed to be sampled from a Gaussian distribution, $\mathcal{N}(0,0.2)$. This system has been used in $[2,3]$ to illustrate theoretical developments for suboptimal dual adaptive control.

In this paper two radial basis function neural networks with 7 and 3 Gaussian basis functions respectively are used to approximate the nonlinear functions $f(y(k))=\sin [y(k)]+\cos [3 y(k)]$ and $g(y(k))=\{2+\cos [y(k)]\}$. The following reference model with input-output pairs $\left\{r(k), y_{m}(k+1)\right\}$ is chosen so that $\mathrm{y}_{\mathrm{m}}(\mathrm{k}+1)$ represents the desired output behavior at time $k+1$

$$
y_{m}(k+1)=r(k)+0.0074 y_{m}(k) .
$$

For comparison purposes, three sets of experiments were conducted to demonstrate the on-line training methods for the proposed Bayesian control algorithms with input-dependent and input-independent hyperparameter and the conventional indirect adaptive control $[16,17]$. On-line adaptation for the parameters of the forward models in the indirect adaptive control method and for the parameters of the forward models and the hyperparameters $\alpha$ and $\beta$ in the Bayesian control method were conducted. The same noise sequence, initial conditions, neural network structure, and reference input were used during implementation of each control method. The result is shown in Figure 1. As expected, the figure shows that the indirect adaptive control exhibits large transient overshoot because it is not taking into consideration the uncertainty of the forward and inverse models. Only after the initial period, when the parameters of the forward and inverse models converge, does the control assume good tracking. On the contrary, and although a different source of uncertainty is introduced (which is mainly coming from the uncertainty introduced from the on-line estimation of the hyperparameters), the Bayesian method with input-independent hyperparameter shows better characteristics in the transient response reflecting the use of knowledge about uncertainty of the forward and inverse models. Besides the better transient performance of the proposed Bayesian method with input-dependent hyperparameter, the steady state performance of this method was much better compared to the classical indirect adaptive control method and the Bayesian method with input-independent hyperparameter. This is also expected and stems from the fact that the noise variance could be different for different choices of input values and therefore its effect on the system output could be significantly reduced. This can also be verified from figure 2 , which shows the probability density function curves of the tracking error at different instants of time. It can be seen from this figure that the pdf of the tracking error becomes deep and narrow after a 
few time steps of control, particulary after time instant 6 indicating that the derived control algorithm decreases the randomness of the tracking error.

\section{Discussion}

Similarly to the method presented in [7], the proposed probabilistic indirect adaptive control in this paper is another Bayes-based control method, but augmented with our prior knowledge on local model approximations and control. To elaborate further, we derive the probabilistic indirect adaptive control with the following points in mind:

- Following the discussion in section 2.3, we assume the dependency of the hyperparameter of the tracking error on the input vector. This ensures consistency with our prior knowledge on local model approximations and control.

- Subsequently, we focus on how to compute the predictive distribution of the system output analytically. This requires Gaussian approximation of the posterior distribution of control signals by Taylor expanding it around its minimum value of $\mathbf{u}_{M P}(k)$ as given in Equation (29). Moreover, since the hyperparameter and the system output models are nonlinear functions of the control signals, they are also approximated by their Taylor expansions about $\boldsymbol{u}_{\mathrm{MP}}(\mathrm{k})$ as given in Equation (32).

- There is no assumption made on whether the process noise has a known probability density function or is a stationary random process. The proposed Bayesian formalism in this paper provides an elegant method for estimating and tracking the noise.

In this line of thinking, the predictive distribution of the system output is shown to have an additional term to the noise variance as can be seen from Equation (36) compared to that derived in [7]. This term is related to the width of the posterior distribution of control signal around the noise variance of the tracking error. This means that via the approach proposed in this paper we have achieved the accurate estimate of the predictive distribution of the system output. Moreover, since functional uncertainties and tracking error noises are considered in the probabilistic adaptive control method proposed in this paper, the derived control law thus possesses robustness. 


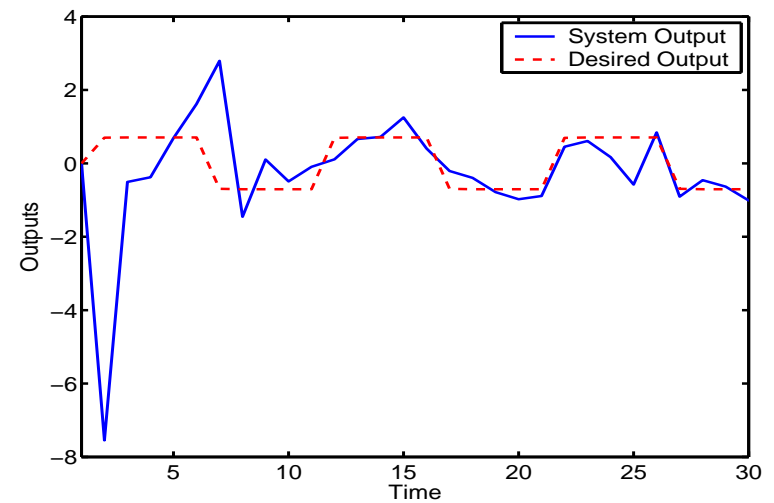

(a)

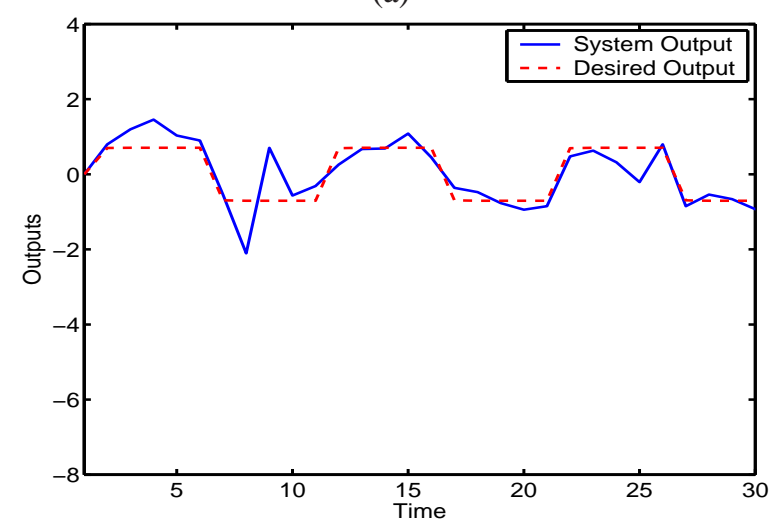

(c)

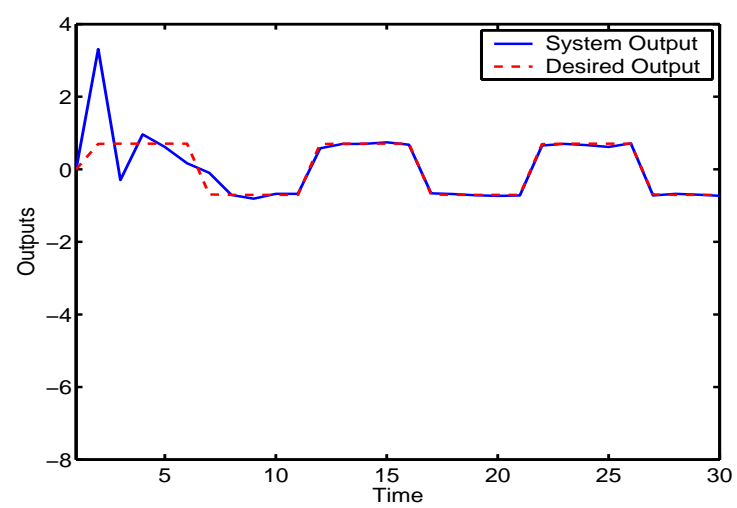

(e)

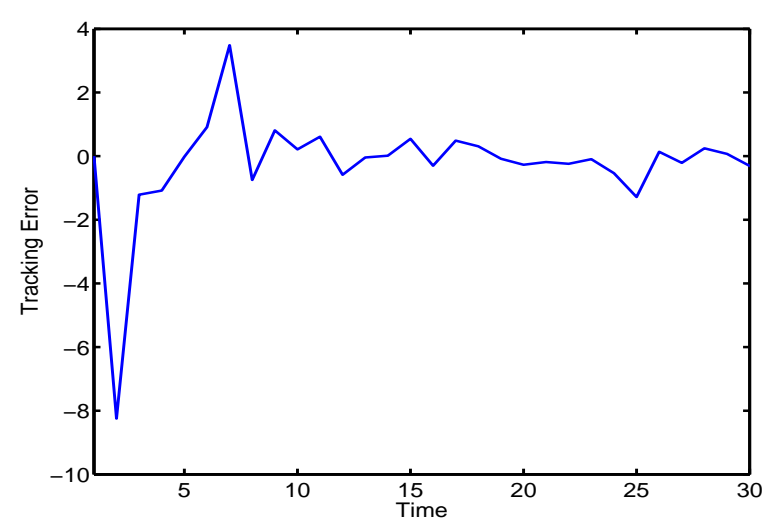

(b)

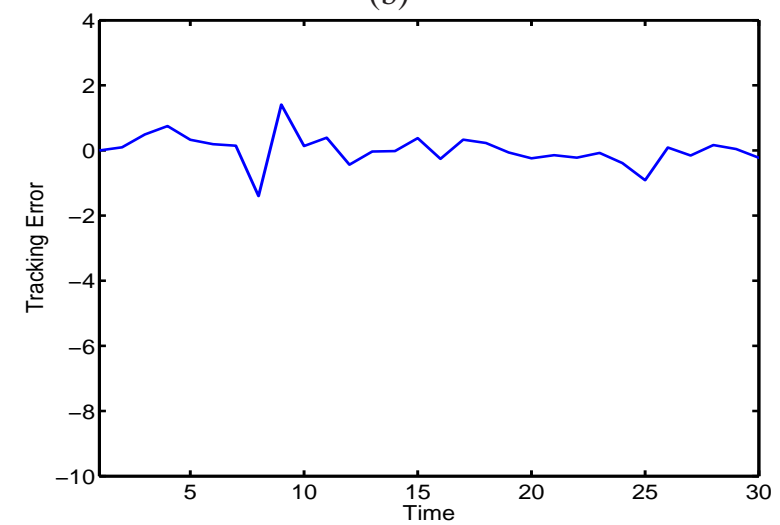

(d)

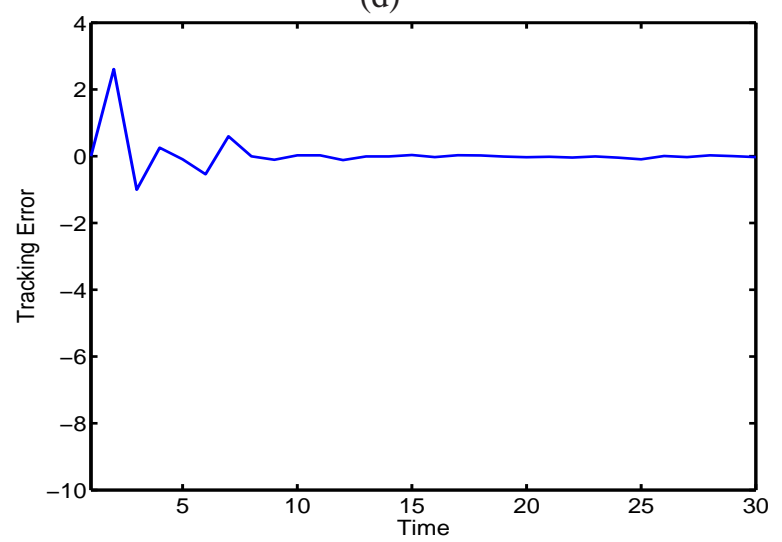

(f)

Figure 1. Results of on-line control: output and tracking error (a) the actual and reference model outputs of standard adaptive control. (b) tracking error of standard adaptive control. (c) the actual and reference model outputs of the proposed Bayesian method with input-independent hyperparameter. (d) tracking error of the proposed Bayesian method with input-independent hyperparameter. (e) the actual and reference model outputs of the proposed Bayesian method with input-dependent hyperparameter. (f) tracking error of the proposed Bayesian method with input-dependent hyperparameter. 


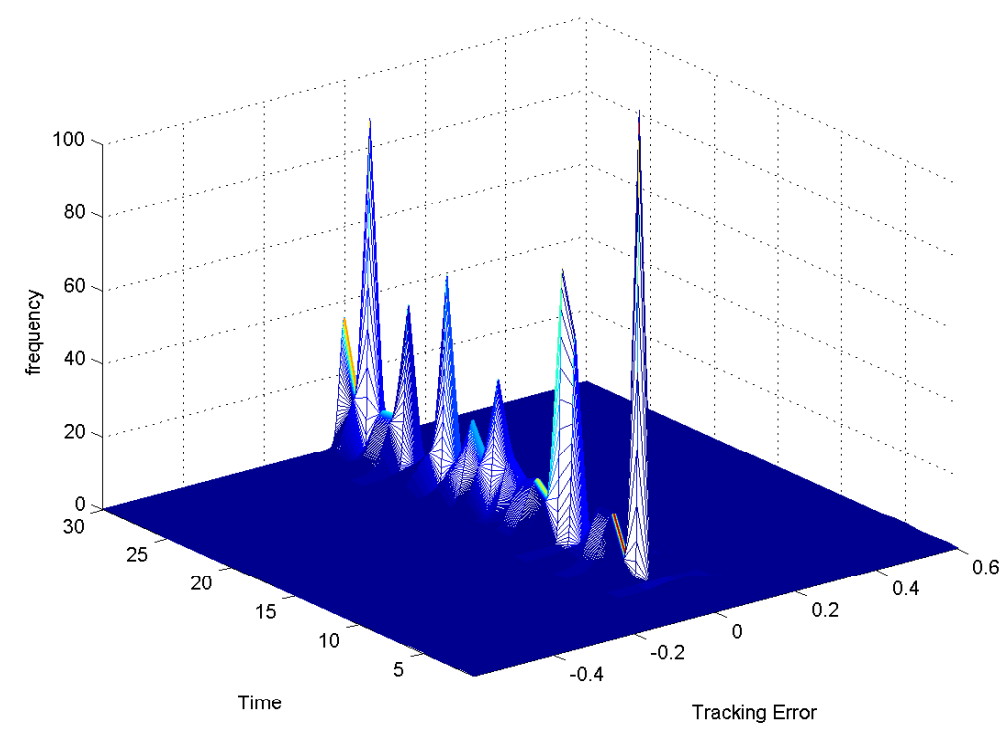

Figure 2. Probability density of tracking error

To sum up, we claim that the proposed probabilistic indirect adaptive control in this paper is more accurate, more principled in mathematical terms and is consistent with local model approximations and control than the method proposed in [7].

The difficulty with the present approach, and ultimately its limitations (as with other nonlinearly optimized control design methods) can be summarized as follows. First, it requires a nonlinear optimization method for the control signal determination. Second, it requires intensive computational load due to the on-line implementation of the method. Third, the stability and closed loop performance are difficult to asses due to the nonlinearity of the dynamics of the process.

Finally, although the Bayesian formalism in this paper is built in the Gaussian domain, it is capable of approximating nonlinear and stochastic systems where a unique control signal can be generated. For stochastic systems characterized by multimodality and Hysteresis, more general distributions of the tracking error and the prior should be considered. Future work will consider how the Gaussian assumption may be relaxed by modeling the required probability density functions using, for example, mixture models. 


\section{Conclusions}

In this paper a new probabilistic indirect adaptive control algorithm for nonlinear systems with inputdependent hyperparameter of tracking error was presented. It provides a theoretical foothold for a wider aim in probabilistic controller design.

Throughout the paper, the basic paradigm for the proposed probabilistic control method has been developed. The discussion then demonstrated the proposed method for general class of nonlinear uncertain discrete time systems where the system equations are taken to be nonlinear functions in both the previous input and output values. Because of the nonlinearity of the system equations a closed form for the optimal control strategy could not be found. The developed method is then applied to the affine class of nonlinear uncertain discrete time systems. The optimal control law is shown to be determined in closed form by solving a cubic equation.

A numerical example demonstrated the improvement due to the inclusion of input-dependent hyperparameter of tracking error. Better transient and steady state performance has been obtained by allowing the dependency of the hyperparameter of the tracking error on the input values.

This more general framework for adaptive control methods has the major advantage that we can now incorporate uncertainty (in models and parameters) in a more structured framework. Different levels of uncertainty and noise models can be treated consistently using the inference machinery. We have chosen to adopt pragmatic and tractable approximations to the general framework, and future work will examine the computational consequences of this proposed methodology.

\section{APPENDIX}

\section{I.1. Gaussian Integral of Input-Dependent Noise}

In this Section the evaluation of the mean and the variance of the following Gaussian integral will be considered 


$$
\begin{aligned}
p(t \mid D) & =\int p(t \mid \mathbf{u}) p(\mathbf{u} \mid D) d u \\
& \propto \int \exp \left[-\frac{1}{2\left[\sigma_{m p}^{2}+q^{\top} \triangle \mathbf{u}+\frac{1}{2} \triangle \mathbf{u}^{\top} M \triangle \mathbf{u}\right]}\left\{t-\left[y_{m}-y_{m p}-g^{\top} \triangle \mathbf{u}\right]\right\}^{2}\right. \\
& \left.-\frac{1}{2} \triangle \mathbf{u}^{\top} A \triangle \mathbf{u}\right] \mathrm{d} \triangle \mathbf{u},
\end{aligned}
$$

where here $\mathrm{y}_{\mathrm{mp}}+\mathrm{g}^{\mathrm{\top}} \triangle \mathbf{u}$ and $\sigma_{\mathrm{mp}}^{2}+\mathrm{q}^{\top} \triangle \mathbf{u}+\frac{1}{2} \triangle \mathbf{u}^{\top} \mathrm{M} \triangle \mathbf{u}$ is the mean and the variance respectively of the distribution $p(t \mid \mathbf{u})$. Let

$$
\begin{gathered}
\mathrm{b}=\mathrm{y}_{\mathrm{m}}-\mathrm{y}_{\mathrm{mp}}-\mathrm{g}^{\top} \triangle \mathbf{u} \\
\mathrm{a}=\frac{1}{\sigma_{\mathrm{mp}}^{2}+\mathrm{q}^{\top} \triangle \mathbf{u}+\frac{1}{2} \triangle \mathbf{u}^{\top} M \triangle \mathbf{u}} .
\end{gathered}
$$

Use Equation (58) in Equation (57), yields

$$
p(t \mid D) \propto \int \exp \left[-\frac{a}{2}(t-b)^{2}-\frac{1}{2} \triangle \mathbf{u}^{\top} A \triangle \mathbf{u}\right] \mathrm{d} \triangle \mathbf{u}
$$

We start firstly by evaluating the mean $\bar{t}$ of the distribution $p(t \mid D)$ by multiplying the integral of Equation (57) by $t$ and integrating over $t$ first and then integrating over $\mathbf{u}$ as follows

$$
\begin{aligned}
\overline{\mathrm{t}} & \propto \iint \mathrm{t} \exp \left[-\frac{\mathrm{a}}{2}(\mathrm{t}-\mathrm{b})^{2}-\frac{1}{2} \triangle \mathbf{u}^{\mathrm{T}} \mathrm{A} \triangle \mathbf{u}\right] \mathrm{d} \triangle \mathbf{u d t} \\
& \propto \iint(\mathrm{t}+\mathrm{b}) \exp \left[-\frac{\mathrm{a}}{2} \mathrm{t}^{2}\right] \mathrm{dt} \exp \left[-\frac{1}{2} \triangle \mathbf{u}^{\mathrm{T}} A \triangle \mathbf{u}\right] \mathrm{d} \triangle \mathbf{u} \\
& =y_{m}-y_{m p} .
\end{aligned}
$$


Next to evaluate the variance $k^{2}$ of the distribution $p(t \mid D)$, we evaluate the second moment of the distribution, integrating over $\mathrm{t}$ first and then integrating over $\mathbf{u}$ as follows

$$
\begin{aligned}
& \bar{t}^{2}+k^{2} \propto \iint t^{2} \exp \left[-\frac{a}{2}(t-b)^{2}-\frac{1}{2} \Delta \mathbf{u}^{\top} A \Delta \mathbf{u}\right] d \Delta \mathbf{u d t} \\
& \propto \iint(t+b)^{2} \exp \left[-\frac{a}{2} t^{2}\right] d t \exp \left[-\frac{1}{2} \Delta \mathbf{u}^{\top} A \triangle \mathbf{u}\right] d \triangle \mathbf{u} \\
& \propto \int\left[\frac{1}{a}+b^{2}\right] \exp \left[-\frac{1}{2} \Delta \mathbf{u}^{\top} A \triangle \mathbf{u}\right] \mathrm{d} \triangle \mathbf{u} \\
& \propto \int[\underbrace{\left(\sigma_{\mathrm{mp}}^{2}+\mathrm{q}^{\top} \Delta \mathbf{u}+\frac{1}{2} \Delta \mathbf{u}^{\top} \mathrm{M} \triangle \mathbf{u}\right)}_{\text {Integral I }} \\
& +\underbrace{\left.\left(y_{m}-y_{m p}-g^{\top} \Delta \mathbf{u}\right)^{2}\right]}_{\text {Integral II }} \exp \left[-\frac{1}{2} \triangle \mathbf{u}^{\top} A \Delta \mathbf{u}\right] \mathrm{d} \triangle \mathbf{u}
\end{aligned}
$$

The evaluation of integral I yields

$$
\begin{aligned}
\text { IntegralI } & \propto \int\left(\sigma_{m p}^{2}+q^{\top} \Delta \mathbf{u}+\frac{1}{2} \Delta \mathbf{u}^{\top} M \triangle \mathbf{u}\right) \exp \left[-\frac{1}{2} \triangle \mathbf{u}^{\top} A \Delta \mathbf{u}\right] \mathrm{d} \triangle \mathbf{u} \\
& =\sigma_{m p}^{2}+\frac{1}{2} \operatorname{Tr}\left(A^{-1} M\right)
\end{aligned}
$$

and the evaluation of integral II yields

$$
\begin{aligned}
\text { IntegralII } & \propto\left[\int\left(y_{m}-y_{m p}\right)^{2} \exp \left[-\frac{1}{2} \triangle \mathbf{u}^{\top} A \triangle \mathbf{u}\right] \mathrm{d} \triangle \mathbf{u}\right. \\
& \left.+\int \triangle \mathbf{u}^{\top} g g^{\top} \triangle \mathbf{u} \exp \left[-\frac{1}{2} \triangle \mathbf{u}^{\top} A \triangle \mathbf{u}\right]\right] \mathrm{d} \triangle \mathbf{u} \\
& =\left(y_{m}-y_{m p}\right)^{2}+g^{\top} A^{-1} g .
\end{aligned}
$$

Adding up the results of integral I and II and equate to the left hand side of Equation (61) while using Equation (60) simultaneously, yields

$$
\begin{aligned}
\kappa^{2}+\left(y_{m}-y_{m p}\right)^{2} & =\left(y_{m}-y_{m p}\right)^{2}+\sigma_{m p}^{2}+g^{\top} A^{-1} g+\frac{1}{2} \operatorname{Tr}\left(A^{-1} M\right) \\
\kappa^{2} & =\sigma_{m p}^{2}+g^{\top} A^{-1} g+\frac{1}{2} \operatorname{Tr}\left(A^{-1} M\right)
\end{aligned}
$$

\section{I.2. Proof of Theorem 2}

In this section we give the proof of Theorem 2 of the re-estimation equations of the hyperparameters of the input-dependent noise model. Here the Hessian $A$ of the error function consists of four terms. 
To unify the definition of the Hessian matrix for the affine and the general classes of nonlinear discrete time systems we rewrite the Hessian matrix here in the form

$$
\mathrm{H}=\mathrm{H}_{1}+\mathrm{H}_{2}+\mathrm{H}_{3}+\alpha \mathrm{I}
$$

where $\mathrm{H}_{1}, \mathrm{H}_{2}, \mathrm{H}_{3}$ correspond to the first, the second and the third term of Equation (30) respectively. We start by considering the problem of finding the maximum of Equation (40) with respect to $\alpha$. If $\left\{\mu_{i}\right\},\left\{\lambda_{i}\right\}$, and $\left\{\vartheta_{i}\right\}$ denote the eigenvalues of $H_{1}, H_{2}, H_{3}$ respectively, then $A$ has eigenvalues $\mu_{i}+\lambda_{i}+\vartheta_{i}+\alpha$ and the derivative of the $\log$ of the Hessian matrix $\ln |A|$ with respect to $\alpha$ is given by

$$
\begin{aligned}
\frac{d}{d \alpha} \ln |A| & =\frac{d}{d \alpha} \ln \left(\Pi_{i}\left(\mu_{i}+\lambda_{i}+\vartheta_{i}+\alpha\right)\right) \\
& =\frac{d}{d \alpha} \sum_{i} \ln \left(\mu_{i}+\lambda_{i}+\vartheta_{i}+\alpha\right) \\
& =\sum_{i} \frac{1}{\mu_{i}+\lambda_{i}+\vartheta_{i}+\alpha}=\operatorname{Tr} A^{-1} .
\end{aligned}
$$

Although the Hessian matrix of the affine class of nonlinear discrete time systems with inputindependent hyperparameter is independent of the control signal, making the variance of the noise of the tracking error a function of the control signal has led to the complication of the Hessian matrix being function of the control signal as could be seen from Equation (54). This implies that as well as for the general nonlinear class of discrete time systems the result given in Equation (66) neglects terms involving $d \lambda / d \alpha$ for the affine class of nonlinear systems.

With this approximation, the maximization of Equation (40) with respect to $\alpha$ leads to the result that at the maximum,

$$
2 \alpha E_{u}^{M P}=r-\sum_{i=1}^{r} \frac{\alpha}{\mu_{i}+\lambda_{i}+\vartheta_{i}+\alpha}=\gamma
$$

where $\gamma$ is defined in Equation (43).

Consider now the maximization of Equation (40) with respect to $\beta$. The derivatives of the eigenvalues $\left\{\mu_{i}\right\},\left\{\lambda_{i}\right\}$, and $\left\{\vartheta_{i}\right\}$ of the Hessian matrices with respect to $\beta$ can be shown to be directly 
proportional to $\beta$ and hence

$$
\begin{aligned}
& \frac{d \mu_{i}}{d \beta}=\frac{2 \mu_{i}}{\beta}, \\
& \frac{d \lambda_{i}}{d \beta}=\frac{3 \lambda_{i}}{\beta}, \\
& \frac{d \vartheta_{i}}{d \beta}=\frac{\vartheta_{i}}{\beta} .
\end{aligned}
$$

Thus the derivative of the $\log$ of the Hessian matrix $\ln |A|$ of the error function with respect to $\beta$ is given by

$$
\begin{aligned}
\frac{d}{d \beta} \ln |A| & =\frac{d}{d \beta} \sum_{i} \ln \left(\mu_{i}+\lambda_{i}+\vartheta_{i}+\alpha\right), \\
& =\frac{1}{\beta} \sum_{i} \frac{2 \mu_{i}+3 \lambda_{i}+\vartheta_{i}}{\mu_{i}+\lambda_{i}+\vartheta_{i}+\alpha} .
\end{aligned}
$$

This leads to the following condition satisfied at the maximum of Equation (40) with respect to $\beta$,

$$
2 \beta E_{E\left[e_{t}\right]}^{M P}=1-\sum_{i=1}^{r} \frac{2 \mu_{i}+3 \lambda_{i}+\vartheta_{i}}{\mu_{i}+\lambda_{i}+\vartheta_{i}+\alpha}=1-\gamma .
$$

Rearranging Equations (67) and (70) yields the re-estimation equations of the hyperparameters $\alpha$ and $\beta$ given by (41) and (42) respectively.

\section{REFERENCES}

1. J. Doyle, B. Francis, and A. Tannenbaum. Feedback Control Theory. Macmillan Publishing Co, Cambridge, Massachusetts, London, England., 1990.

2. S. Fabri and V. Kadirkamanathan. Dual adaptive control of nonlinear stochastic systems using neural networks. Automatica, 34(2):245-253, February 1998.

3. S. G. Fabri and V. Kadirkamanathan. Functional Adaptive Control: An Intelligent Systems Approach. Springer-Verlag, February 2001.

4. A. Gameiro. Baud sampling bit synchroniser for channels with data dependent noise. Electronics Letters, 34(21):20002002, 1998.

5. R. Herzallah and D. Lowe. Robust control of nonlinear stochastic systems by modelling conditional distributions of control signals. Neural Computing and Applications, 12:98-108, 2003.

6. R. Herzallah and D. Lowe. Distribution modeling of nonlinear inverse controllers under a Bayesian framework. IEEE Transactions on Neural Networks, 18(1):107-114, January 2007.

7. R. Herzallah and D. Lowe. A Bayesian perspective on stochastic neuro control. IEEE Transactions on Neural Networks, 19(5):914-924, May 2008. 
8. Y. Ho and R. Lee. A bayesian approach to problems in stochastic estimation and control. IEEE Transactions on Automatic Control, 9(4):333-339, October 1964.

9. M. Kárný. Towards fully probabilistic control design. Automatica, 32(12):1719-1722, 1996.

10. R. J. Kornblum and M. Tribus. The use of bayesian inference in the design of an endpoint control system for the basic oxygen steel furnace. IEEE Transactions on Systems Science and Cybernetics, 6(4):339-348, October 1970.

11. X. R. Li and Y. Bar-Shalom. A recursive multiple model approach to noise identification. IEEE Transactions on Aerospace Electronic Systems, 30(3):671-684, July 1994.

12. D. J. C. Mackay. Bayesian interpolation. Neural Computation, 4:415-447, 1992.

13. P. Maybeck. Stochastic Models, Estimation and Control, volume 2. Academic Press, New York, 1982.

14. R. Mehra. Approaches to adaptive filtering. IEEE Transactions on Automatic Control, 17(5):693-698, October 1972.

15. W. T. Miller, R. S. Sutton, and P. J. Werbos, editors. Neural Networks for Control. MIT Press, Cambridge, Massachusetts, London, England., 1990.

16. K. S. Narendra. Adaptive control using neural networks. In D. A. White and D. Sofge, editors, Handbook of Intelligent Control, chapter 5, pages 141-183. Multiscience Press, Inc, New York, 1992.

17. K. S. Narendra and S. Mukhopadhyay. Adaptive control of nonlinear multivariable systems using neural networks. Neural Networks, 7(5):737-752, 1994.

18. R. W. D. Nickalls. A new approach to solving the cubic: Cardan's solution revealed. The Mathematical Gazette, 77:354359,1993

19. Simo Särkkä and Aapo Nummenmaa. Recursive noise adaptive kalman filtering by variational bayesian approximations. IEEE Transactions on Automatic Control, 54(3):596-600, March 2009.

20. Shiliang Sun, Changshui Zhang, and Guoqiang Yu. A bayesian network approach to traffic flow forecasting. IEEE Transactions on Intelligent Transportation Systems, 7(1):124-132, March 2006.

21. Magalie Thomassin, Thierry Bastogne, and Alain Richard. Identification of a managed river reach by a bayesian approach. IEEE Transactions on Automatic Control, 17(2):353-365, March 2009.

22. H. Wang. Minimum entropy control of non-gaussian dynamic stochastic systems. IEEE Transactions on Automatic Control, 47(2):398-403, 2002.

23. H. Wang and J. Zhang. Bounded stochastic distribution control for pseudo armax stochastic systems. IEEE Transactions on Automatic Control, 46(3):486-490, 2001. 